\title{
Study of Solubility of Atorvastatin using Ternary Phase Diagram for the Development of Self-Emulsifying Drug Delivery Systems (SEDDS)
}

\author{
Fariba Khan ${ }^{1}$, Md. Saiful Islam ${ }^{2}$ and Reza-ul J aliI ${ }^{2}$ \\ ${ }^{1}$ Department of Pharmacy, The University of Asia Pacific, Dhanmondi, Dhaka-1209, Bangladesh. \\ ${ }^{2}$ Department of Pharmaceutical Technology, Faculty of Pharmacy, University of Dhaka, Dhaka-1000, \\ Bangladesh.
}

\begin{abstract}
Self-emulsifying drug delivery system (SEDDS) is successfully used to improve the aqueous solubility and oral bioavailability of the poorly aqueous soluble drugs. Atorvastatin calcium (ATV), a poorly aqueous soluble drug having low oral bioavailability, was the model drug for this study. The aim of this study was to find out the suitable lipid and surfactant which can be used in formulation of ATV-SEDDS and this was done using ternary phase diagram, an important tool used very essentially in optimizing SEDDS formulations. Ternary phase diagrams of lipid/surfactant/ATV mixture were constructed to generate the solubility data of ATV. Two lipids namely Capmul PG 8, Oleic acid and seven different surfactants namely Tween 20, Tween 80, Cremophor CO 40, Cremophor CO 60, Cremophor EL, Cremophor RH 40 and Cremophor RH 60 were used. For Capmul PG 8/surfactant mixture, solubilizing efficiency order was: Cremophor RH $40>$ Tween $80>$ Tween $20>$ Cremophor CO $60>$ Cremophor RH $60>$ Cremophor EL $>$ Cremophor CO 40. For Oleic acid/surfactant mixture, solubilizing efficiency order was: Cremophor RH $40>$ Tween $80>$ Tween $20>$ Cremophor RH $60>$ Cremophor CO $60>$ Cremophor EL $>$ Cremophor $\mathrm{CO} 40$. Considering the solubility phase diagrams of the drug, both Oleic acid and Capmul PG 8 can be used as lipid in combination with any of the surfactants, Cremophor RH40 or Tween 80 or Tween 20 for the development of SEDDS formulations of ATV having enhanced solubility and dissolution property.
\end{abstract}

Kew words: Atorvastatin calcium, Ternary phase diagram, Self-emulsifying drug delivery system

\section{INTRODUCTION}

Atorvastatin calcium (ATV), a 3-hydroxy-3methylglutaryl coenzyme A (HMG-CoA) reductase inhibitor, is a plasma lipid regulating agent. ATV has therapeutic applications in hyperlipidemia and cardiovascular events.

$\operatorname{ATV}\left(\left[\mathrm{R}-\left(\mathrm{R}, \mathrm{R}^{*}\right)-2-\right.\right.$ (4-fluorophenyl)- $\beta, \gamma$-dihydroxy-5- (1-methylethyl) -3-phenyl-4- (phenylamino) carbonyl]-1H-pyrrol-1-heptanoic acid, hemi-calcium salt) is a white to off-white crystalline powder. The empirical formula of atorvastatin calcium is $\left(\mathrm{C}_{33} \mathrm{H}_{34} \mathrm{FN}_{2} \mathrm{O}_{5}\right)_{2} \mathrm{Ca}^{2+} \cdot 3 \mathrm{H}_{2} \mathrm{O}$ with a molecular weight of 1209.42 . The oral bioavailability of atorvastatin is limited by factors such as the membrane permeability, the solubility, the dissolution rate of the

Correspondence to: Md. Saiful Islam

$+88-01732073108$ (mobile)

E-mail: msipharmacy@du.ac.bd, saif_phdu@yahoo.com

Dhaka Univ. J. Pharm. Sci. 11(2): 83-91, 2012 (December) drug and so on. Specially, the solubility and the dissolution rate of a sparingly water soluble drug is a critical factor for its oral bioavailability. Oral bioavailability of ATV is only $12 \%$ and its poor solubility in water and high presystemic clearance $(>$ $80 \%$ ) have been attributed to its poor bioavailability. ${ }^{1}$

According to the USP, very slightly soluble drugs are defined as solubility value of $0.1 \mathrm{mg} / \mathrm{ml}$ to $1 \mathrm{mg} / \mathrm{ml}$; insoluble or practically insoluble drugs are those having a solubility value of $<0.1 \mathrm{mg} / \mathrm{ml}(100$ $\mu \mathrm{g} / \mathrm{ml})$. According to biopharmaceutical classification system (BCS), low solubility means drug will not dissolve in $250 \mathrm{~mL}$ of buffer solution throughout the $\mathrm{pH}$ range of 1 to 8 . According to Lindenberg et al. (2004), ${ }^{2}$ low solubility means dissolution time of dose will be greater than normal transit time through normal absorption regions of GI tract.

ATV is insoluble in aqueous solution of $\mathrm{pH} \leq 4.0$ and below; it is very slightly soluble in water and 
slightly soluble at $\mathrm{pH} 7.4$ phosphate buffers and acetonitrile, slightly soluble in ethanol and freely soluble in methanol. The intestinal permeability of ATV is high at the physiologically intestinal $\mathrm{pH}$ of 6.0-6.5. ${ }^{1}$

The purpose of this present study was to investigate and generate the solubility data of ATV in different lipid/surfactant mixtures where lipid/surfactant ratios were permutated to generate the maximum solubility data. Ternary Phase Diagram was used to present the solubility profile of the drug where amount of atorvastatin, lipid and surfactant were the three variables.

\section{MATERIALS AND METHODS}

Atorvastatin calcium was purchased from Ranbaxy, India. Oleic acid (Merck, Germany), Capmul PG 8 (Abitec Corporation, USA), Tween 20 and 80 (BDH Chemicals Ltd, England) was received as gift. Cremophor CO 40, Cremophor CO 60, Cremophor EL, Cremophor RH 40, Cremophor RH 60 were also received as gifts from BASF (BASF, Germany).

Solubility study of atorvastatin in different excipients. Mixtures of lipids (Capmul PG 8 or Oleic acid) and surfactants (Tween 20, Tween 80, Cremophor CO 40, Cremophor CO 60, Cremophor EL, Cremophor RH 40, Cremophor RH 60) at different ratios were prepared in captubes. Briefly, ATV was gradually added in each of the captube up to the maximum solubility capacity of lipid/surfactant mixture. After each increment, the captube containing lipid/surfactant/drug mixture was heated in sealed condition in a water bath at $\leq 90^{\circ} \mathrm{C}$ for $\leq 5$ minutes to facilitate the solubilization of ATV. Solubility points (percentage of lipid, surfactant and ATV) were then plotted in a ternary phase diagram. Few of the major points in the ternary phase diagram considered during solubility study and their corresponding result are shown in table 1-14. After connecting the solubility points in the phase diagram, a soluble area of ATV was found.

\section{RESULTS AND DISCUSSION}

This research study was conducted with the aim of generating some solubility data of ATV in lipid/surfactant mixture. In the ternary phase diagram, individual point $(\mathrm{X}, \mathrm{Y}, \mathrm{Z})$ representing the weight percentage of lipid, drug, and surfactant was considered to check whether atorvastatin is soluble/insoluble at that particular point. Only the major solubility points of the drug and those, near the soluble/insoluble boundary area of the diagram, are shown in table 1-14.

Table 1. Solubility data of atorvastatin in Capmul PG 8Cremophor EL mixture

\begin{tabular}{lcccl}
\hline $\begin{array}{l}\text { Points } \\
\text { No. }\end{array}$ & $\begin{array}{c}\text { \% of } \\
\text { Capmul } \\
\text { PG } 8 \\
\text { (wt/wt) }\end{array}$ & $\begin{array}{c}\text { \% of } \\
\text { Cremo- } \\
\text { phor EL } \\
\text { (wt/wt) }\end{array}$ & $\begin{array}{c}\text { \% of } \\
\text { Atorvas- } \\
\text { tatin } \\
\text { (wt/wt) }\end{array}$ & Remarks \\
\hline I & 99 & 0 & 1 & Soluble \\
II & 90 & 0 & 10 & Soluble \\
III & 80 & 10 & 10 & Soluble \\
IV & 70 & 20 & 10 & Soluble \\
V & 60 & 30 & 10 & Soluble \\
VI & 50 & 40 & 10 & Soluble \\
VII & 40 & 50 & 10 & Soluble \\
VIII & 30 & 60 & 10 & Soluble \\
IX & 20 & 70 & 10 & Soluble \\
X & 10 & 80 & 10 & Soluble \\
XI & 0 & 99 & 1 & Soluble \\
XII & 60 & 20 & 20 & Insoluble \\
XIII & 0 & 80 & 20 & Insoluble \\
XIV & 40 & 40 & 20 & Insoluble \\
XV & 80 & 0 & 20 & Insoluble \\
\hline
\end{tabular}

Table 2. Solubility data of atorvastatin in Oleic acidCremophor EL mixture

\begin{tabular}{lcccl}
\hline $\begin{array}{l}\text { Points } \\
\text { No. }\end{array}$ & $\begin{array}{c}\text { \% of } \\
\text { Oleic } \\
\text { Acid } \\
\text { (wt/wt) }\end{array}$ & $\begin{array}{c}\text { \% of } \\
\text { Cremo- } \\
\text { phor EL } \\
\text { (wt/wt) }\end{array}$ & $\begin{array}{c}\text { \% of } \\
\text { Atorvas- } \\
\text { tatin } \\
\text { (wt/wt) }\end{array}$ & Remarks \\
\hline I & 99 & 0 & 1 & Soluble \\
II & 90 & 0 & 10 & Soluble \\
III & 80 & 0 & 20 & Soluble \\
IV & 70 & 0 & 30 & Soluble \\
V & 80 & 10 & 10 & Soluble \\
VI & 70 & 20 & 10 & Soluble \\
VII & 70 & 10 & 20 & Soluble \\
VIII & 60 & 30 & 10 & Soluble \\
IX & 60 & 20 & 20 & Soluble \\
X & 50 & 40 & 10 & Soluble \\
XI & 40 & 50 & 10 & Soluble \\
XII & 30 & 60 & 10 & Soluble \\
XIII & 20 & 70 & 10 & Soluble \\
XIV & 10 & 80 & 10 & Soluble \\
XV & 0 & 90 & 10 & Soluble \\
XVI & 30 & 50 & 20 & Insoluble \\
XVII & 0 & 80 & 20 & Insoluble \\
XVIII & 40 & 40 & 20 & Insoluble \\
\hline & & & & \\
\hline
\end{tabular}


Table 3. Solubility data of atorvastatin in Capmul PG8Cremophor CO 40 mixture

\begin{tabular}{lcccl}
\hline $\begin{array}{l}\text { Points } \\
\text { No. }\end{array}$ & $\begin{array}{c}\text { \% of } \\
\text { Capmul } \\
\text { PG } 8 \\
(\mathrm{wt} / \mathrm{wt})\end{array}$ & $\begin{array}{c}\text { \% of } \\
\text { Cremopho } \\
\text { r CO 40 } \\
(\mathrm{wt} / \mathrm{wt})\end{array}$ & $\begin{array}{c}\text { \% of } \\
\text { Atorvastat } \\
\text { in (wt/wt) }\end{array}$ & Remarks \\
\hline I & 99 & 0 & 1 & Soluble \\
II & 90 & 0 & 10 & Soluble \\
III & 80 & 0 & 20 & Soluble \\
IV & 70 & 0 & 30 & Soluble \\
V & 80 & 10 & 10 & Soluble \\
VI & 70 & 20 & 10 & Soluble \\
VII & 70 & 10 & 20 & Insoluble \\
VIII & 60 & 30 & 10 & Soluble \\
IX & 60 & 20 & 20 & Insoluble \\
X & 50 & 40 & 10 & Soluble \\
XI & 40 & 50 & 10 & Soluble \\
XII & 30 & 60 & 10 & Soluble \\
XIII & 20 & 70 & 10 & Soluble \\
XIV & 10 & 80 & 10 & Soluble \\
XV & 0 & 90 & 10 & Insoluble \\
XVI & 30 & 50 & 20 & Insoluble \\
XVII & 0 & 80 & 20 & Insoluble \\
XVIII & 40 & 40 & 20 & Insoluble \\
\hline & & & & \\
\hline
\end{tabular}

Table 4. Solubility data of atorvastatin in Oleic acidCremophor CO 40 mixture

\begin{tabular}{lcccl}
\hline $\begin{array}{l}\text { Points } \\
\text { No. }\end{array}$ & $\begin{array}{c}\text { \% of } \\
\text { Oleic } \\
\text { Acid } \\
\text { (wt/wt) }\end{array}$ & $\begin{array}{c}\text { \% of } \\
\text { Cremopho } \\
\text { r CO 40 } \\
\text { (wt/wt) }\end{array}$ & $\begin{array}{c}\text { \% of } \\
\text { Atorvastat } \\
\text { in (wt/wt) }\end{array}$ & Remarks \\
\hline I & 99 & 0 & 1 & Soluble \\
II & 90 & 0 & 10 & Soluble \\
III & 80 & 0 & 20 & Soluble \\
IV & 70 & 0 & 30 & Soluble \\
V & 80 & 10 & 10 & Soluble \\
VI & 70 & 20 & 10 & Soluble \\
VII & 70 & 10 & 20 & Soluble \\
VIII & 60 & 30 & 10 & Soluble \\
IX & 60 & 20 & 20 & Soluble \\
X & 50 & 40 & 10 & Soluble \\
XI & 40 & 50 & 10 & Soluble \\
XII & 30 & 60 & 10 & Soluble \\
XIII & 20 & 70 & 10 & Soluble \\
XIV & 10 & 80 & 10 & Insoluble \\
XV & 0 & 90 & 10 & Insoluble \\
XVI & 30 & 50 & 20 & Insoluble \\
XVII & 0 & 80 & 20 & Insoluble \\
XVIII & 40 & 40 & 20 & Insoluble \\
\hline
\end{tabular}

In the solubility phase diagrams, darker regions indicate soluble area for ATV. Two lipids (Capmul PG 8 and Oleic acid) and seven different surfactants of two particular groups (polyoxyethylene castor oil derivatives and polyoxyethylene sorbitan fatty acid esters) were used.

Table 5. Solubility data of atorvastatin in Capmul PG8Cremophor CO 60 mixture

\begin{tabular}{lcccl}
\hline $\begin{array}{l}\text { Points } \\
\text { No. }\end{array}$ & $\begin{array}{c}\text { \% of } \\
\text { Capmul } \\
\text { PG8 } \\
(\mathrm{wt} / \mathrm{wt})\end{array}$ & $\begin{array}{c}\text { \% of } \\
\text { Cremopho } \\
\text { r CO 60 } \\
\text { (wt/wt) }\end{array}$ & $\begin{array}{c}\text { \% of } \\
\text { Atorvastat } \\
\text { in (wt/wt) }\end{array}$ & Remarks \\
\hline I & 99 & 0 & 1 & Soluble \\
II & 90 & 0 & 10 & Soluble \\
III & 80 & 0 & 20 & Insoluble \\
IV & 70 & 0 & 30 & Insoluble \\
V & 80 & 10 & 10 & Soluble \\
VI & 70 & 20 & 10 & Soluble \\
VII & 70 & 10 & 20 & Insoluble \\
VIII & 60 & 30 & 10 & Soluble \\
IX & 60 & 20 & 20 & Insoluble \\
X & 50 & 40 & 10 & Soluble \\
XI & 40 & 50 & 10 & Soluble \\
XII & 30 & 60 & 10 & Soluble \\
XIII & 20 & 70 & 10 & Soluble \\
XIV & 10 & 80 & 10 & Soluble \\
XV & 0 & 90 & 10 & Soluble \\
XVI & 30 & 50 & 20 & Insoluble \\
XVII & 0 & 80 & 20 & Soluble \\
XVIII & 40 & 40 & 20 & Insoluble \\
\hline & & & &
\end{tabular}

Table 6. Solubility data of atorvastatin in Oleic acidCremophor CO 60 mixture

\begin{tabular}{lcccl}
\hline $\begin{array}{l}\text { Points } \\
\text { No. }\end{array}$ & $\begin{array}{c}\text { \% of } \\
\text { Oleic } \\
\text { Acid } \\
\text { (wt/wt) }\end{array}$ & $\begin{array}{c}\text { \% of } \\
\text { Cremopho } \\
\text { r CO 60 } \\
(w t / w t)\end{array}$ & $\begin{array}{c}\text { \% of } \\
\text { Atorvastat } \\
\text { in (wt/wt) }\end{array}$ & Remarks \\
\hline I & 99 & 0 & 1 & Soluble \\
II & 90 & 0 & 10 & Soluble \\
III & 80 & 0 & 20 & Soluble \\
IV & 70 & 0 & 30 & Soluble \\
V & 80 & 10 & 10 & Soluble \\
VI & 70 & 20 & 10 & Soluble \\
VII & 70 & 10 & 20 & Soluble \\
VIII & 60 & 30 & 10 & Soluble \\
IX & 60 & 20 & 20 & Soluble \\
X & 50 & 40 & 10 & Soluble \\
XI & 40 & 50 & 10 & Soluble \\
XII & 30 & 60 & 10 & Soluble \\
XIII & 20 & 70 & 10 & Soluble \\
XIV & 10 & 80 & 10 & Soluble \\
XV & 0 & 90 & 10 & Soluble \\
XVI & 30 & 50 & 20 & Insoluble \\
XVII & 0 & 80 & 20 & Insoluble \\
XVIII & 40 & 40 & 20 & Insoluble \\
\hline & & & &
\end{tabular}

Figures 1-7 show the ternary phase diagrams for ATV solubility. Figure 1 shows the solubility 
diagram for Cremophor EL. Better solubility was achieved while this surfactant was mixed with oleic acid. Figure 2 shows the solubility diagram of Cremophor $\mathrm{CO}$ 40. In this case also, oleic acid/Cremophor CO 40 mixture showed better solubility than Capmul PG 8/Cremophor CO 40 mixture. Similarly, figure 3 , figure 4 , figure 5 , figure 6 and figure 7 show the solubility diagram of ATV where surfactants were Cremophor CO 60, Cremophor RH 40, Cremophor RH 60, Tween 20 and Tween 80 respectively. Though oleic acid/surfactant mixture showed better solubility than Capmul PG 8/surfactant mixture, both Oleic acid and Capmul PG 8 dissolved remarkable amount of ATV (8-35\% $\mathrm{wt} / \mathrm{wt})$. Particularly, Cremophor RH $40 \quad \approx 35 \%$ $\mathrm{wt} / \mathrm{wt})$ and Tween $80(\approx 30 \% \mathrm{wt} / \mathrm{wt})$ showed the best solubility profile in combination with both lipid components. These results are also in accordance with the solubility data reported by Talegaonkar et al. (2010) and Shen and Zhong (2006). ${ }^{3,4}$

Table 7. Solubility data of atorvastatin in Capmul PG8Cremophor RH 40 mixture

\begin{tabular}{lcccl}
\hline $\begin{array}{l}\text { Points } \\
\text { No. }\end{array}$ & $\begin{array}{c}\text { \% of } \\
\text { Capmul } \\
\text { PG8 } \\
\text { (wt/wt) }\end{array}$ & $\begin{array}{c}\text { \% of } \\
\text { Cremopho } \\
\text { r RH 40 } \\
\text { (wt/wt) }\end{array}$ & $\begin{array}{c}\text { \% of } \\
\text { Atorvastat } \\
\text { in (wt/wt) }\end{array}$ & Remarks \\
\hline I & 90 & 0 & 10 & Soluble \\
II & 80 & 0 & 20 & Insoluble \\
III & 80 & 10 & 10 & Soluble \\
IV & 70 & 10 & 20 & Insoluble \\
V & 70 & 20 & 10 & Soluble \\
VI & 60 & 30 & 10 & Soluble \\
VII & 60 & 20 & 20 & Insoluble \\
VIII & 50 & 40 & 10 & Soluble \\
IX & 50 & 30 & 20 & Insoluble \\
X & 40 & 50 & 10 & Soluble \\
XI & 40 & 40 & 20 & Soluble \\
XII & 30 & 50 & 20 & Soluble \\
XIII & 10 & 60 & 30 & Soluble \\
XIV & 0 & 60 & 40 & Insoluble \\
XV & 0 & 64 & 36 & Soluble \\
XVI & 20 & 50 & 30 & Insoluble \\
\hline
\end{tabular}

A wide variety of lipids are available for the development of oral lipid-based formulations including long chain and medium chain triglycerides, propylene glycol esters, mono and diglycerides of medium chain and long chain fatty acids, various lipid mixtures, and so on. ${ }^{5,6}$ Adding to the diversity, the fatty acid components of the lipids can be either saturated or unsaturated, varying the field even more. According to Cannon and Long $(2008)^{6}$, lipids that

Table 8. Solubility data of atorvastatin in Oleic acidCremophor RH 40 mixture

\begin{tabular}{lcccl}
\hline $\begin{array}{l}\text { Points } \\
\text { No. }\end{array}$ & $\begin{array}{c}\text { \% of } \\
\text { Oleic } \\
\text { Acid } \\
\text { (wt/wt) }\end{array}$ & $\begin{array}{c}\text { \% of } \\
\text { Cremopho } \\
\text { r RH 40 } \\
\text { (wt/wt) }\end{array}$ & $\begin{array}{c}\text { \% of } \\
\text { Atorvastat } \\
\text { in (wt/wt) }\end{array}$ & Remarks \\
\hline I & 90 & 0 & 10 & Soluble \\
II & 80 & 0 & 20 & Soluble \\
III & 70 & 0 & 30 & Soluble \\
IV & 64 & 0 & 36 & Soluble \\
V & 60 & 0 & 40 & Insoluble \\
VI & 60 & 10 & 30 & Soluble \\
VII & 50 & 10 & 40 & Insoluble \\
VIII & 50 & 20 & 30 & Soluble \\
IX & 40 & 20 & 40 & Insoluble \\
X & 40 & 30 & 30 & Soluble \\
XI & 30 & 30 & 40 & Insoluble \\
XII & 30 & 40 & 30 & Soluble \\
XIII & 0 & 60 & 40 & Insoluble \\
XIV & 10 & 60 & 30 & Soluble \\
XV & 0 & 64 & 36 & Soluble \\
\hline & & & & \\
\hline
\end{tabular}

Table 9. Solubility data of atorvastatin in Capmul PG 8Cremophor RH 60 mixtures

\begin{tabular}{lcccl}
\hline $\begin{array}{l}\text { Points } \\
\text { No. }\end{array}$ & $\begin{array}{c}\text { \% of } \\
\text { Capmul } \\
\text { PG8 } \\
(\mathrm{wt} / \mathrm{wt})\end{array}$ & $\begin{array}{c}\text { \% of } \\
\text { Cremopho } \\
\text { r RH } 60 \\
(\mathrm{wt} / \mathrm{wt})\end{array}$ & $\begin{array}{c}\text { \% of } \\
\text { Atorvastat } \\
\text { in (wt/wt) }\end{array}$ & Remarks \\
\hline I & 90 & 0 & 10 & Soluble \\
II & 80 & 0 & 20 & Insoluble \\
III & 80 & 10 & 10 & Soluble \\
IV & 70 & 20 & 10 & Soluble \\
V & 70 & 10 & 20 & Insoluble \\
VI & 60 & 30 & 10 & Soluble \\
VII & 60 & 20 & 20 & Insoluble \\
VIII & 50 & 40 & 10 & Soluble \\
IX & 50 & 30 & 20 & Insoluble \\
X & 40 & 50 & 10 & Soluble \\
XI & 40 & 40 & 20 & Insoluble \\
XII & 30 & 60 & 10 & Soluble \\
XIII & 30 & 50 & 20 & Insoluble \\
XIV & 20 & 70 & 10 & Soluble \\
XV & 20 & 60 & 20 & Insoluble \\
XVI & 10 & 80 & 10 & Soluble \\
XVII & 10 & 70 & 20 & Insoluble \\
XVIII & 0 & 80 & 20 & Soluble \\
XIX & 0 & 79 & 21 & Insoluble \\
\hline & & & & \\
\hline & & & & \\
\hline
\end{tabular}


Table 10. Solubility data of atorvastatin in Oleic acidCremophor RH 60 mixtures

\begin{tabular}{lcccl}
\hline $\begin{array}{l}\text { Points } \\
\text { No. }\end{array}$ & $\begin{array}{c}\text { \% of } \\
\text { Oleic acid } \\
\text { (wt/wt) }\end{array}$ & $\begin{array}{c}\text { \% of } \\
\text { Cremopho } \\
\text { r RH 60 } \\
(\mathrm{wt} / \mathrm{wt})\end{array}$ & $\begin{array}{c}\text { \% of } \\
\text { Atorvastat } \\
\text { in (wt/wt) }\end{array}$ & Remarks \\
\hline I & 90 & 0 & 10 & Soluble \\
II & 80 & 0 & 20 & Soluble \\
III & 70 & 0 & 30 & Soluble \\
IV & 60 & 0 & 40 & Insoluble \\
V & 67 & 33 & 0 & Soluble \\
VI & 60 & 10 & 30 & Soluble \\
VII & 50 & 20 & 30 & Soluble \\
VIII & 40 & 30 & 30 & Insoluble \\
IX & 40 & 40 & 20 & Soluble \\
X & 30 & 40 & 30 & Insoluble \\
XI & 30 & 50 & 20 & Soluble \\
XII & 20 & 50 & 30 & Insoluble \\
XIII & 20 & 60 & 20 & Soluble \\
XIV & 10 & 60 & 30 & Insoluble \\
XV & 10 & 70 & 20 & Soluble \\
XVI & 0 & 70 & 30 & Insoluble \\
XVII & 0 & 80 & 20 & Soluble \\
\hline
\end{tabular}

Table 11. Solubility data of atorvastatin in Capmul PG8Tween 20 mixtures

\begin{tabular}{lcccl}
\hline $\begin{array}{l}\text { Points } \\
\text { No. }\end{array}$ & $\begin{array}{c}\text { \% of } \\
\text { Capmul } \\
\text { PG8 } \\
\text { (wt/wt) }\end{array}$ & $\begin{array}{c}\text { \% of } \\
\text { Tween 20 } \\
\text { (wt/wt) }\end{array}$ & $\begin{array}{c}\text { \% of } \\
\text { Atorvastat } \\
\text { in (wt/wt) }\end{array}$ & Remarks \\
\hline I & 90 & 0 & 10 & Soluble \\
II & 80 & 0 & 20 & Insoluble \\
III & 80 & 10 & 10 & Soluble \\
IV & 70 & 10 & 20 & Insoluble \\
V & 70 & 20 & 10 & Soluble \\
VI & 60 & 20 & 20 & Insoluble \\
VII & 60 & 30 & 10 & Soluble \\
VIII & 50 & 30 & 20 & Insoluble \\
IX & 50 & 40 & 10 & Soluble \\
X & 40 & 40 & 20 & Insoluble \\
XI & 40 & 50 & 10 & Soluble \\
XII & 30 & 50 & 20 & Insoluble \\
XIII & 30 & 60 & 10 & Soluble \\
XIV & 20 & 60 & 20 & Soluble \\
XV & 10 & 70 & 20 & Soluble \\
XVI & 0 & 80 & 20 & Soluble \\
XVII & 0 & 70 & 30 & Insoluble \\
XVIII & 0 & 73 & 27 & Soluble \\
\hline
\end{tabular}

have fatty acid chains of 14-20 carbons are considered long chain, while those with 6-12 carbons are medium chain. Unless they consist of unsaturated fatty acid chains, the long-chain glycerides are usually solid at room temperature and, therefore, may

Table 12. Solubility data of atorvastatin in Oleic acid-Tween 20 mixtures

\begin{tabular}{lcccl}
\hline $\begin{array}{l}\text { Points } \\
\text { No. }\end{array}$ & $\begin{array}{c}\text { \% of } \\
\text { Oleic acid } \\
\text { (wt/wt) }\end{array}$ & $\begin{array}{c}\text { \% of } \\
\text { Tween } 20 \\
\text { (wt/wt) }\end{array}$ & $\begin{array}{c}\text { \% of } \\
\text { Atorvastat } \\
\text { in (wt/wt) }\end{array}$ & Remarks \\
\hline I & 90 & 0 & 10 & Soluble \\
II & 80 & 0 & 20 & Soluble \\
III & 70 & 0 & 30 & Soluble \\
IV & 60 & 0 & 40 & Insoluble \\
V & 65 & 0 & 35 & Soluble \\
VI & 60 & 10 & 30 & Soluble \\
VII & 50 & 20 & 30 & Soluble \\
VIII & 40 & 30 & 30 & Soluble \\
IX & 30 & 40 & 30 & Insoluble \\
X & 30 & 42 & 28 & Soluble \\
XI & 20 & 50 & 30 & Insoluble \\
XII & 20 & 60 & 20 & Soluble \\
XIII & 10 & 60 & 30 & Insoluble \\
XIV & 10 & 70 & 20 & Soluble \\
XV & 0 & 70 & 30 & Insoluble \\
XVI & 0 & 73 & 27 & Soluble \\
\hline
\end{tabular}

Table 13. Solubility data of atorvastatin in Capmul PG8Tween 80 mixtures

\begin{tabular}{lcccl}
\hline $\begin{array}{l}\text { Points } \\
\text { No. }\end{array}$ & $\begin{array}{c}\text { \% of } \\
\text { Capmul } \\
\text { PG8 } \\
(\mathrm{wt} / \mathrm{wt})\end{array}$ & $\begin{array}{c}\text { \% of } \\
\text { Tween } 80 \\
(\mathrm{wt} / \mathrm{wt})\end{array}$ & $\begin{array}{c}\text { \% of } \\
\text { Atorvastat } \\
\text { in (wt/wt) }\end{array}$ & Remarks \\
\hline I & 90 & 0 & 10 & Soluble \\
II & 80 & 0 & 20 & Insoluble \\
III & 70 & 20 & 10 & Soluble \\
IV & 70 & 10 & 20 & Insoluble \\
V & 60 & 20 & 20 & Insoluble \\
VI & 60 & 21 & 19 & Soluble \\
VII & 50 & 30 & 20 & Soluble \\
VIII & 40 & 30 & 30 & Insoluble \\
IX & 40 & 40 & 20 & Soluble \\
X & 30 & 40 & 30 & Insoluble \\
XI & 30 & 50 & 20 & Soluble \\
XII & 20 & 50 & 30 & Insoluble \\
XIII & 20 & 60 & 20 & Soluble \\
XIV & 10 & 60 & 30 & Insoluble \\
XV & 10 & 70 & 20 & Soluble \\
XVI & 0 & 70 & 30 & Soluble \\
XVII & 0 & 60 & 40 & Insoluble \\
XVIII & 0 & 68 & 32 & Soluble \\
\hline & & & &
\end{tabular}

not be suitable for dissolving drugs. Further, longchain glycerides which exist as liquids at room temperature (e.g., corn oil, sesame oil, peanut oil, olive oil, soybean oil, etc.) have been reported to have lower drug solubilities than medium-chain 
Table 14. Solubility data of atorvastatin in Oleic acid-Tween 80 mixtures

\begin{tabular}{lcccl}
\hline $\begin{array}{l}\text { Points } \\
\text { No. }\end{array}$ & $\begin{array}{c}\text { \% of } \\
\text { Oleic } \\
\text { Acid } \\
(\mathrm{wt} / \mathrm{wt})\end{array}$ & $\begin{array}{c}\text { \% of } \\
\text { Tween } 80 \\
(\mathrm{wt} / \mathrm{wt})\end{array}$ & $\begin{array}{c}\% \text { of } \\
\text { Atorvastat } \\
\text { in (wt/wt) }\end{array}$ & Remarks \\
\hline I & 90 & 0 & 10 & Soluble \\
II & 80 & 0 & 20 & Soluble \\
III & 70 & 0 & 30 & Soluble \\
IV & 60 & 0 & 40 & Insoluble \\
V & 65 & 0 & 35 & Soluble \\
VI & 60 & 10 & 30 & Soluble \\
VII & 50 & 20 & 30 & Soluble \\
VIII & 40 & 30 & 30 & Soluble \\
IX & 30 & 40 & 30 & Soluble \\
X & 20 & 50 & 30 & Soluble \\
XI & 10 & 60 & 30 & Soluble \\
XII & 0 & 70 & 30 & Insoluble \\
XIII & 0 & 71 & 29 & Soluble \\
\hline
\end{tabular}
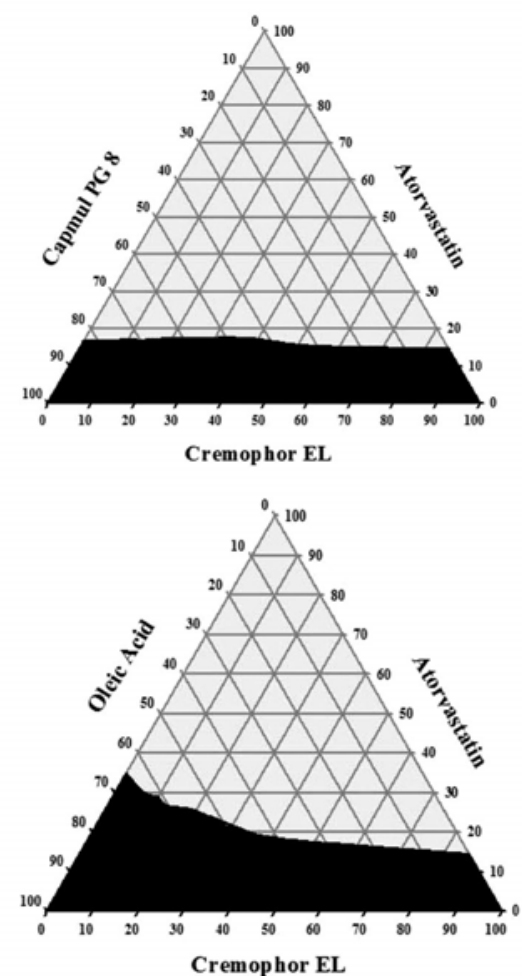

Figure 1. Solubility phase diagram of ATV using Cremophor EL

glycerides. ${ }^{7,8}$ Modified or hydrolyzed vegetable oils have been widely used since these excipients form good emulsification system with a large number of surfactants approved for oral administration and exhibit better drug solubility properties. ${ }^{9-11}$ But natural long chain derivatives like oleic acid, which can be defined as amphiphilic compounds with
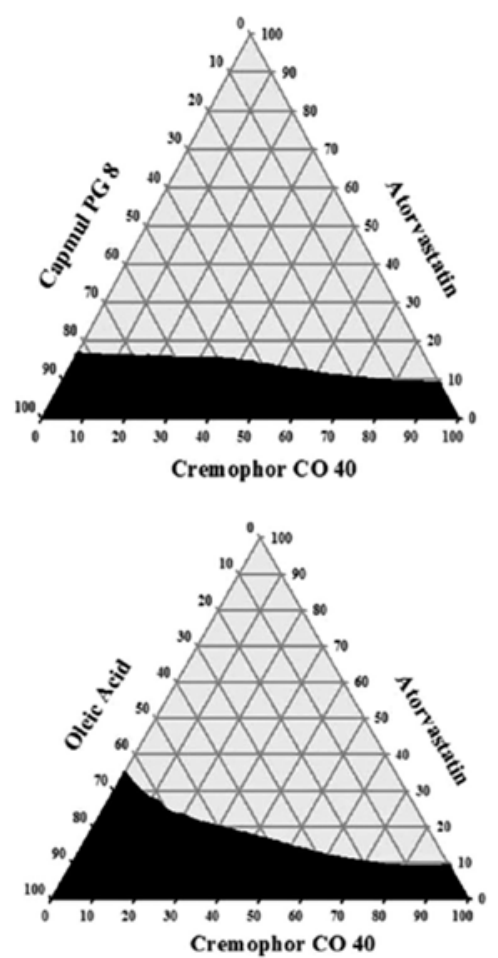

Figure 2. Solubility phase diagram of ATV using Cremophor CO 40
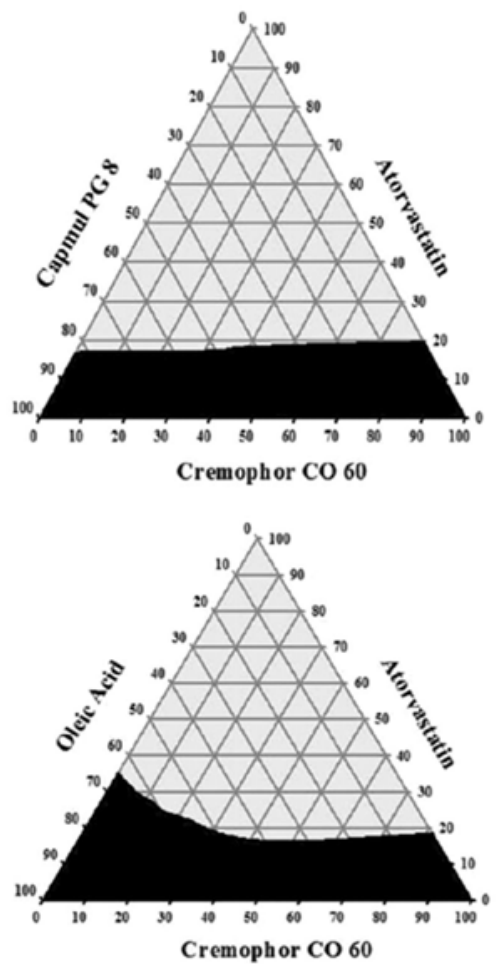

Figure 3. Solubility phase diagram of ATV using Cremophor CO 60 

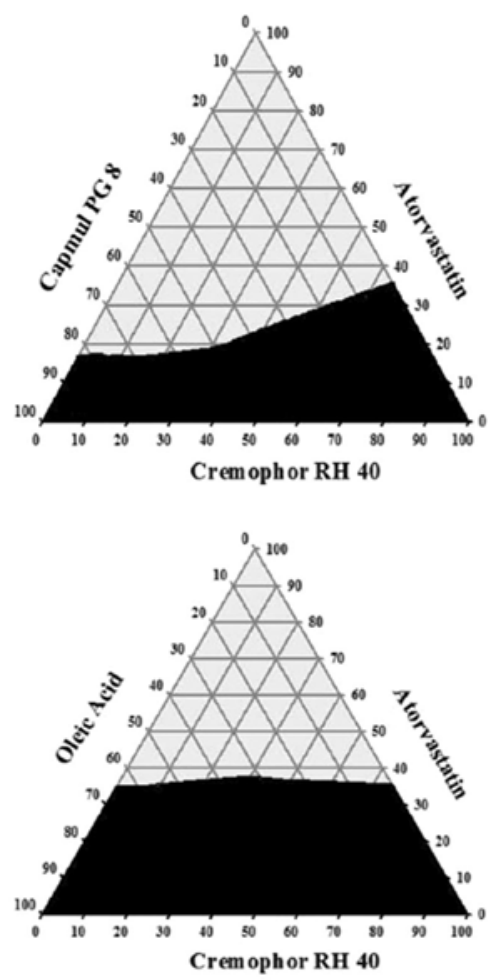

Figure 4. Solubility phase diagram of ATV using Cremophor RH 40
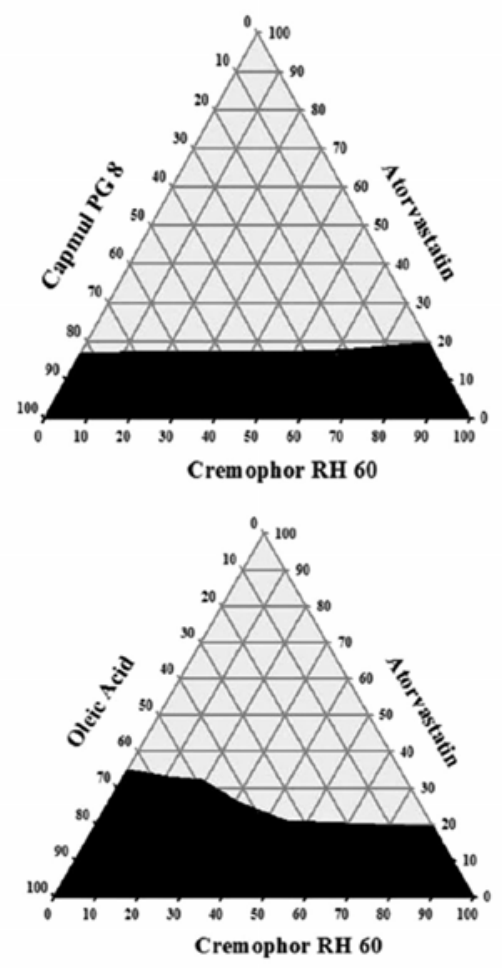

Figure 5. Solubility phase diagram of ATV using Cremophor RH 60
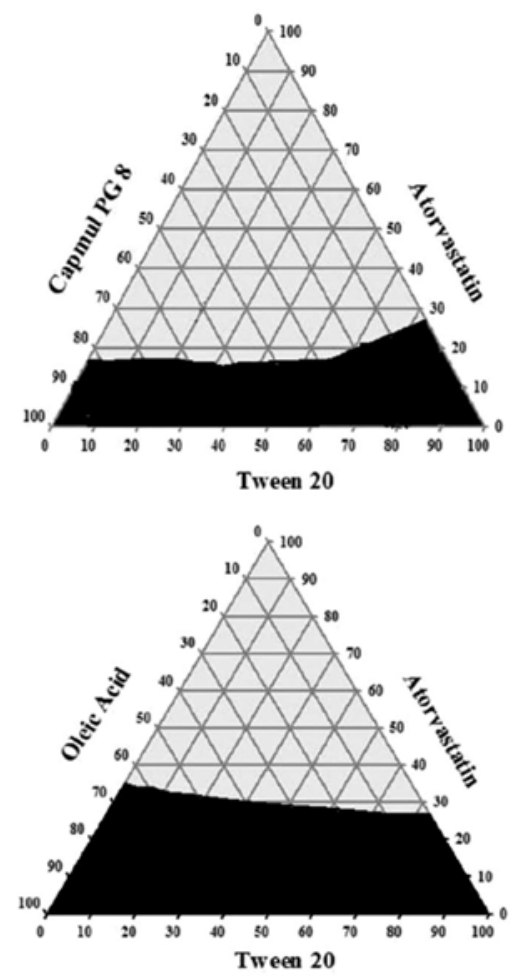

Figure 6. Solubility phase diagram of ATV using Tween 20
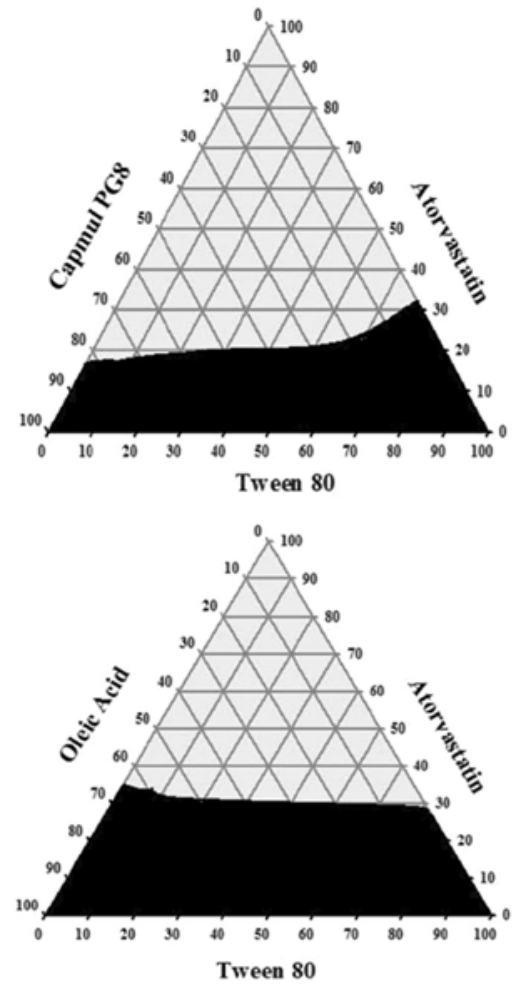

Figure 7. Solubility phase diagram of ATV using Tween 80 
surfactant properties, are progressively and effectively replacing the regular medium chain triglyceride lipids in the self-emulsifying formulations (SEFs). ${ }^{9,12,13}$ The second lipid compound was Capmul PG 8 which is a propylene glycol monoester or propylene glycol monocaprylate of $\mathrm{C} 8$ fatty acids. It is a synthetic medium chain lipid and is a good choice of excipient in oral-lipid based formulations. Due to its lipophilic hydrophiliclipophilic balance (HLB) value of 5.0 and evidences of successful application in SEFs, ${ }^{14,15}$ it was considered to be used as lipid component in our study. As part of an ongoing research to optimizing some novel SEDDS formulations, it was tried to find out the best combination of lipid-surfactant mixture with respect to maximum ATV solubility since lipid/surfactant/drug ratio plays the pivotal role in the development of SEDDS. ${ }^{16}$

However, more than $15 \%$ ATV was found to be dissolved in case of all the combinations of lipid/surfactant mixture. Amount of ATV dissolved in either single excipient or in combination of lipid/surfactant was found as maximum as $18 \%$ (wt/wt) for Cremophor EL and Cremophor CO 40, $20 \%$ (wt/wt) for Cremophor CO 60 and Cremophor RH $60,30 \%$ (wt $/ w t$ ) for Tween 20 , up to $35 \%$ (wt/wt) for Tween 80 and Cremophor RH 40. It indicates that the ATV solubilizing efficiency order of the selected surfactants in combination with Capmul PG 8 was: Cremophor RH $40>$ Tween $80>$ Tween $20>$ Cremophor CO $60>$ Cremophor RH 60 $>$ Cremophor EL > Cremophor CO 40. On the other hand, solubilizing capacity order of the selected surfactants in combination with Oleic acid was: Cremophor RH $40>$ Tween $80>$ Tween $20>$ Cremophor RH $60>$ Cremophor CO $60>$ Cremophor EL $>$ Cremophor CO 40. It can be inferred that Cremophor RH 40 showed maximum solubilizing capacity of ATV followed by Tween 80 and Tween 20.

Non-ionic surfactants with a relatively high HLB values are the most widely recommended surfactants for SEFs. Cremophor grades are the nonionic solubilizers and emulsifying agents obtained by reacting hydrogenated castor oil with ethylene oxide. Though, Cremophor $\mathrm{CO}$ grades are the cosmetic grades, Cremophor RH comply according to The European Pharmacopoeia/The United States Pharmacopoeia (EP/USP). ${ }^{17}$ Both of the Cremophor RH grades showed good solubilizing capacity for ATV. But, RH 40 was better than RH 60. Chemically RH 40 is a glycerol polyethyleneglycol oxystearate which, together with fatty acid glycerol polyglycol esters, forms the hydrophobic part of the product. The hydrophilic part consists of polyethylene glycols and glycerol ethoxylated. It is a non-ionic solubilizer and emulsifying agent having a HLB value of 14.016.0 and a $\mathrm{pH}$ value of 6-7. ${ }^{17}$ Due to this non-ionic hydrophilic property and a favorable $\mathrm{pH}$ value, both of which attribute to ATV solubilization, Cremophor RH 40 is a good choice of emulsifier for oral lipid based formulations. ${ }^{4,18}$ Among the non-ionic hydrophilic polysorbates, Tween 80 (polyoxyethylene 20 oleate) is the most widely used one. Due to its hydrophilic HLB value of 15.0, a good number of research works have been reported about the suitability, efficiency and compatibility of Tween 80 for using it in oral lipid based formulations..$^{9,19-22}$

\section{CONCLUSION}

It can be inferred from the obtained results that atorvastatin solubility may be improved by using different lipid/non-ionic hydrophilic surfactants where the lipid/surfactant ratio should be maintained at the optimum level. It has been shown by the ternary phase diagram that either Oleic acid or Capmul PG 8 can be a good choice of lipid in oral lipid-based formulations where either of the Cremophor RH grades (Cremophor RH 40 and Cremophor RH 60) or Tween grades (Tween 20, Tween 80) may be used as the surfactant.

\section{ACKNOWLEDGEMENTS}

Authors would like to thank BASF, Bangladesh and Rangs Pharmaceuticals Ltd., Bangladesh for providing the raw materials. 


\section{REFERENCE}

1. Corsini, A., Bellosta, S., Baetta, R., Fumagalli, R., Paoletti, R. and Bernini, F. 1999. New insights into the pharmacodynamic and pharmacokinetic properties of statins. Pharmacol. Ther. 84, 413-428.

2. Lindenberg, M., Kopp, S. and Dressman, J.B. 2004. Classification of orally administered drugs on the World Health Organization model list of essential medicines according to the biopharmaceutics classification system. Eur. J. Pharm. Biopharm. 58, 265-78.

3. Talegaonkar, S., Mustafa, G., Akhter, S. and Iqbal, Z.I. 2010. Design and development of oral oil-in-water nanoemulsion formulation bearing atorvastatin: in vitro assessment. $J$. Disper. Sci. Tech. 31, 690-701.

4. Shen, H. and Zhong, M. 2006. Preparation and evaluation of self-microemulsifying drug delivery systems (SMEDDS) containing atorvastatin. J. Pharm. Pharmacol. 58, 11831191.

5. Mullertz, A., Ogbonna, A., Ren, S. and Rades, T. 2010. New perspectives on lipid and surfactant based drug delivery systems for oral delivery of poorly soluble drugs. J. Pharm. Pharmacol. 62, 1622-1636.

6. Cannon, J.B. and Long, M.A. 2008. Emulsions, microemulsions, and lipid-based drug delivery systems for drug solubilization and delivery-Part II: Oral applications, in Liu R., (ed) Water-Insoluble Drug Formulation. $2^{\text {nd }}$ ed., CRC Press, Boca Raton, FL, pp. 227-253.

7. Li, P., Hynes, S.R., Haefele, T.F., Pudipeddi, M., Royce, A.E. and Serajuddin, A.T.M. 2009. Development of clinical dosage forms for a poorly water-soluble drug II: Formulation and characterization of a novel solid microemulsion preconcentrate system for oral delivery of a poorly watersoluble drug. J. Pharm. Sci. 5, 1750-1764.

8. Land, L.M., Li, P. and Bummer, P.M. 2005. The influence of water content of triglyceride lipids on the solubility of steroids. Pharm. Res. 22, 784-788.

9. Constantinides, P.P. 1995. Lipid microemulsions for improving drug dissolution and oral absorption: physical and biopharmaceutical aspects. Pharm. Res. 12, 1561-72.

10. Kimura, M., Shizuki, M., Miyoshi, K., Sakai, T., Hidaka, H., Takamura, H. and Matoba, T. 1994. Relationship between the molecular structures and emulsification properties of edible oils. Biosci. Biotech. Biochem. 58, 1258-61.

11. Hauss, D.J., Fogal, S.E., Ficorith, J.V., Price, C.A., Roy, T., Jayaraj, A.A. and Keirns, J.J. 1998. Lipid-based delivery systems for improving the bioavailability and lymphatic transport of a poorly water soluble $\mathrm{LTB}_{4}$ inhibitor. J. Pharm. Sci. 87, 164-9.
12. Karim, A., Gokhale, R., Cole, M., Sherman, J., Yeramian, P., Bryant, M. and Franke, H. 1994. HIV protease inhibitor SC52151: a novel method of optimizing bioavailability profile via a microemulsion drug delivery systems. Pharm. Res. 11, S368.

13. Kibbe, A.H. 2000. Handbook of Pharmaceutical Excipients. $3^{\text {rd }}$ Ed.; p. 356.

14. Prajapati, H.N., Dalrymple, D.M. and Serajuddin, A.T. 2012. A Comparative evaluation of mono-, di-, and triglyceride of medium chain fatty acids by oil/surfactant/water phase diagram, solubility determination and dispersion testing for application in pharmaceutical dosage form development. Pharm. Res. 29, 285-305.

15. Technical Information of Capmul ${ }^{\circledR}$, ABITEC, USA.

16. Prajapati, H.N., Patela, D.P., Patela, N.G., Dalrympleb, D.M. and Serajuddin, A.T.M. 2011. Effect of difference in fatty acid chain lengths of medium-chain oils on oil/surfactant/water phase diagrams and drug solubility. $J$. Excipients Food Chem. 2, 73-88.

17. Technical Information of Cremophor Grades, BASF, Germany.

18. Nielsen, F.S., Gibault, E., Ljusberg-Wahren, H., Arleth, L., Pedersen, J.S. and Anette, M. 2007. Characterization of prototype self-nanoemulsifying formulations of lipophilic compounds. J. Pharm. Sci. 96, 876-92.

19. Dong-Qin, Q., Gui-Xia, X. and Xiang-Gen, W. 2007. Formulation development and pharmacokinetics of puerarin self-emulsifying drug delivery systems. PDA J. Pharm. Sci. Technol. 61, 37-43.

20. Patel, A.R. and Vavia, P.R. 2007. Preparation and In Vivo Evaluation of SMEDDS (Self-Microemulsifying Drug Delivery System) containing fenofibrate. The AAPS J. 9, 34452.1 .

21. Zhao, Y., Wang, C., Chow, A.H.L, Ren, K., Gong, T., Zhang, Z. and Zheng, Y. 2010. Self-nanoemulsifying drug delivery system (SNEDDS) for oral delivery of zedoary essential oil: formulation and bioavailability studies. Int. J. Pharmaceutics. 383, 170-177.

22. Technical Information of Polysorbates, Merck KGaA, Darmstadt, Germany. 